\title{
Control of interference effects in a two-dimensional-electron-gas/superconductor junction by the Josephson effect
}

\author{
Th. Schäpers, ${ }^{*}$ R. P. Müller ${ }^{\dagger}$ and A. Kaluza ${ }^{\ddagger}$ \\ Institut für Schichten und Grenzflächen, Forschungszentrum Jülich, 52425 Jülich, Germany
}

(Received 11 December 2002; published 28 February 2003)

\begin{abstract}
The effect of quasiparticle interferences on the transport between a two-dimensional electron gas in an $\mathrm{In}_{x} \mathrm{Ga}_{1-x} \mathrm{As} / \mathrm{InP}$ heterostructure and the superconducting $\mathrm{Nb}$ electrodes of a Josephson junction is studied. The phase difference between the superconducting electrodes leading to the interference effects is adjusted by the Josephson effect. The quasiparticle interference results in a conductance plateau with a maximum at zero bias current through the Josephson junction. By applying a magnetic field the interference effect can be controlled.
\end{abstract}

DOI: 10.1103/PhysRevB.67.060502

A combination of normal conductors with superconducting electrodes opened up the possibility to discover interesting new effects based on Andreev reflection. ${ }^{1-3}$ In an Andreev reflection process an electron that travels from the normal conductor side on a superconductor/normalconductor ( $\mathrm{SN}$ ) interface is retroreflected as a hole. On the superconductor side a Cooper pair is created simultaneously. When a second superconductor electrode is added so that one ends up with a SNS sandwich, a Josephson current due to phase-coherent Andreev reflection can be observed. As for Josephson tunnel junctions the phase difference $\phi$ between the superconducting electrodes can be adjusted by the supercurrent. ${ }^{4}$

If a normal conductor is sandwiched between two superconducting electrodes, the resistance between the normal conductor and one of the superconducting electrodes is a periodic function of the phase difference $\phi$ between the superconducting electrodes. ${ }^{5-18}$ In all experiments cited above, the difference of the macroscopic phase was controlled by threading a magnetic flux through a superconducting loop with a gap connected by a normal conductor. Alternatively, the phase difference between two superconducting electrodes can also be adjusted by means of the Josephson effect, ${ }^{19}$ i.e., for an ideal Josephson tunnel junction the phase difference is related to the supercurrent by $I_{s}=I_{c} \sin \phi$, where $I_{c}$ is the critical current. Based on this mechanism Nakano and Takayanagi ${ }^{20}$ proposed an interferometer setup with the two branches of the normal conductor connected to the superconducting electrodes of a Josephson tunnel junction.

In this Communication, we discuss quasiparticle interference effects between a normal conductor and the superconducting electrodes of a SNS junction. In contrast to the $e x$ ternal control of the interference by a magnetic flux in a superconducting loop, the phase difference between the superconducting electrodes is controlled internally by the Josephson effect. As a normal conductor we chose a twodimensional electron gas (2DEG) in an $\mathrm{In}_{x} \mathrm{Ga}_{1-x} \mathrm{As} / \mathrm{InP}$ heterostructure. It will be shown that the conductance between the 2DEG and one of the superconducting electrodes is controlled by the phase difference induced by the Josephson bias current flowing through the $S / 2 \mathrm{DEG} / S$ junction. A maximum is found at zero bias current due to constructive interference. By applying a magnetic field the local phase difference between the electrodes is modified leading to a shift of the maximum.
PACS number(s): 74.50. $+\mathrm{r}, 73.23 .-\mathrm{b}$
The 2DEG is realized by using an $\operatorname{In}_{x} \mathrm{Ga}_{1-x} \mathrm{As} / \mathrm{InP}$ layer system grown by metal-organic vapor phase epitaxy. The heterostructure consists of a 300-nm thick InP buffer layer, a 9-nm thick $n$-doped $\operatorname{InP}(\mathrm{Si})$ layer, a 20 -nm thick InP spacer, followed by a 10 -nm thick strained $\operatorname{In}_{0.77} \mathrm{Ga}_{0.23}$ As channel layer, and a 115 -nm thick $\mathrm{In}_{0.53} \mathrm{Ga}_{0.47} \mathrm{As}$ cap layer. From magneto-transport measurements at $0.6 \mathrm{~K}$ a sheet electron concentration of $7.68 \times 10^{11} \mathrm{~cm}^{-2}$ and a mobility of $363600 \mathrm{~cm}^{2} / \mathrm{V}$ s were deduced.

A schematic illustration of the sample layout is shown in Fig. 1(a). A semiconductor mesa was defined by electron beam lithography and reactive ion etching $\left(\mathrm{CH}_{4} / \mathrm{H}_{2}\right)$. The etching depth of $200 \mathrm{~nm}$ was well below the depth of the channel layer. The width of the semiconductor mesa used as a normal conductor bridge was $d=500 \mathrm{~nm}$. As ohmic contacts to the 2DEG Ni/Au:Ge/Ni pads were used. For the fab-

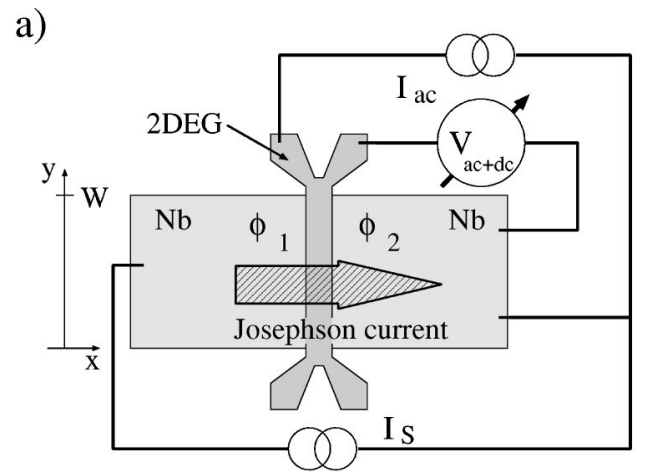

b)

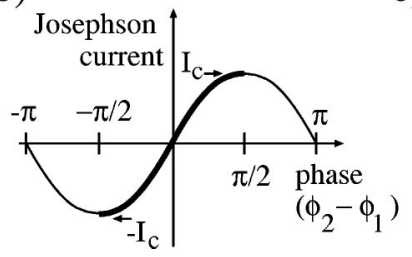

c)

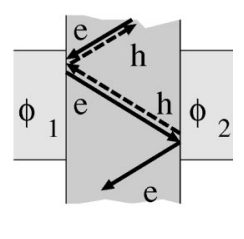

FIG. 1. (a) Illustration of the sample layout and the measurement configuration. The width of the $\mathrm{Nb}$ electrodes is $W=6 \mu \mathrm{m}$. The separation is $500 \mathrm{~nm}$. (b) Sinusoidal current-phase relation of an ideal Josephson junction. The bold line shows the range accessible by biasing the junction from $-I_{c}$ to $+I_{c}$. (c) Electron and hole trajectories for a single point of reflection at each $S / 2 \mathrm{DEG}$ interface. 


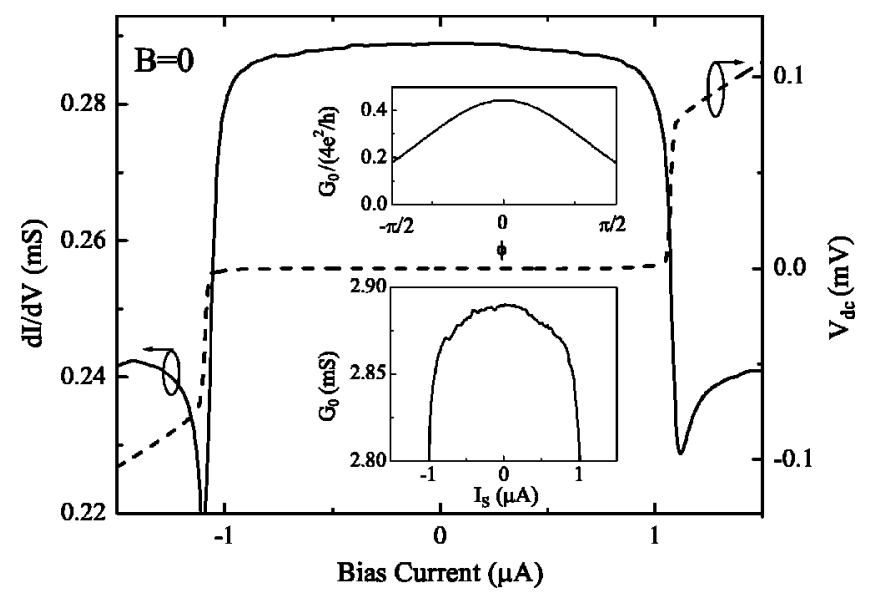

FIG. 2. (a) Differential conductance $d I / d V$ and voltage drop between the upper normal electrode and the right-hand $\mathrm{Nb}$ electrode as a function of the junction bias current at $B=0$ for $T=0.6 \mathrm{~K}$. The lower inset shows a detail of the differential conductance curve. In the upper inset the calculated zero-bias conductance for a single is plotted as a function of the phase difference.

rication of the superconducting electrodes of the $S / 2 \mathrm{DEG} / S$ junction a 100-nm thick $\mathrm{Nb}$ layer was deposited by electron beam evaporation. Prior to the deposition, the sample surface was cleaned by means of a He electron cyclotron resonance plasma source. ${ }^{21}$ The geometry of the Nb electrodes was defined by the electron beam lithography and reactive ion etching $\left(\mathrm{SF}_{6}\right)$. The width of the $\mathrm{Nb}$ electrodes was $W=6 \mu \mathrm{m}$. The superconducting electrodes make contact to the $2 \mathrm{DEG}$ at the sidewalls of the semiconductor mesa. The Nb layer had a critical temperature of $8.0 \mathrm{~K}$.

The Josephson current in the $S / 2 \mathrm{DEG} / S$ junction inducing the phase difference $\phi$ is supplied by a dc current source [Fig. 1(a)]. The conductance between the 2DEG and the right-hand $\mathrm{Nb}$ electrode was measured by using a lock-in technique with an excitation current $I_{a c}=10 \mathrm{nA}$ at a frequency of $173 \mathrm{~Hz}$. All measurements were performed at a temperature of $0.6 \mathrm{~K}$.

In order to study the interference effects between the normal conductor and the junction, we first have to find the range of $S / 2 \mathrm{DEG} / S$ junction bias current, where the junction is in the superconductive state. Only then, the voltage drop at the $S / 2 \mathrm{DEG}$ interfaces is zero and a well-defined timeindependent phase difference $\phi=\phi_{1}-\phi_{2}$ between both $\mathrm{Nb}$ electrodes can be expected. Figure 2 (dashed line) shows the corresponding measurement of the dc voltage drop between the 2DEG and the right-hand $\mathrm{Nb}$ electrode as a function of the $S / 2 \mathrm{DEG} / S$ junction bias current $I_{s}$ at zero magnetic field $(B=0)$. In between $\pm 1.1 \mu \mathrm{A}$ zero voltage $\left(V_{d c}=0\right)$ is measured at the interface. In this range the junction is in the Josephson state, as confirmed by the measurement of the critical current $I_{c}$ of the $S / 2 \mathrm{DEG} / S$ junction. If the junction is in the resistive state, $V_{d c}$ corresponds to about half the value measured at the complete $S / 2 \mathrm{DEG} / S$ junction.

As can be seen in Fig. 2, the conductance between the 2DEG and the superconducting electrodes depends on the bias current $I_{S}$ through the $S / 2 \mathrm{DEG} / S$ junction. Here, the transport properties are measured by driving a small ac cur- rent $I_{a c}$ between the 2DEG and the right-hand side Nb electrode and by detecting the corresponding ac voltage drop $V_{a c}$. The differential conductance $d I / d V$ is determined from $I_{a c} / V_{a c}$. It exhibits a pronounced plateau if the $S / 2 \mathrm{DEG} / S$ is in the Josephson state. For $I_{s}$ larger than $I_{c}$ the differential conductance is lower by about $20 \%$.

We will now focus on the range of bias currents where the $S / 2 \mathrm{DEG} / S$ junction is in the superconducting state $\left(\left|I_{s}\right|<I_{c}\right)$. In this case no voltage drop $\left(V_{d c}=0\right)$ between the 2DEG and the superconducting electrode occurs so that the zero-bias conductance $G_{0}$ is detected between the 2DEG and the superconductor. The transport properties directly at the Fermi energy are accessed. Although $V_{d c}=0$, the differential conductance $G_{0}$ varies if the Josephson current $I_{s}$ is changed. As can be seen in Fig. 2 (lower inset), $G_{0}$ has its maximum at $I_{s}=0$, while it decreases if the Josephson supercurrent is increased. At $B=0$ the $G_{0}$ vs $I_{s}$ curve is symmetric.

In what follows we will show that the dependence of $G_{0}$ on $I_{s}$ can be explained by interference effects in the spirit of the interferometer proposed by Nakano and Takayanagi. ${ }^{20}$ If a supercurrent flows through the $S / 2 \mathrm{DEG} / S$ junction, a phase difference $\phi$ is built up between the superconducting electrodes. In Fig. 1(b) the sinusoidal current-phase relation of an ideal Josephson tunnel junction is depicted schematically. It is known that for the SNS weak link structures the currentphase relation can deviate from this ideal behavior. ${ }^{22}$ However, from independent transport measurements on single $S / 2$ DEG interfaces we could deduce that a potential barrier exists at the interface. ${ }^{23}$ Numerical simulations of a $S / 2 \mathrm{DEG} / S$ junction showed that due to the barrier and the finite junction width the current-phase relation is well approximated by the sinusoidal dependence. Thus, the range of $\phi$ is approximately given by $[-\pi / 2, \pi / 2]$ if the supercurrent is varied from $-I_{c}$ to $+I_{c}$, as illustrated in Fig. 1(b) (bold line).

From the values of the electron concentration and the mobility an elastic mean free path of $l_{e}=5.3 \mu \mathrm{m}$ is deduced. This value is considerably larger than the separation of the $\mathrm{Nb}$ electrodes. It can thus be expected that the transport between the electrodes is close to the ballistic regime. In the interferometer proposed by Nakano and Takayanagi ${ }^{20}$ a beam splitter was employed to obtain two paths for the interfering particles, which is not exactly the configuration of our sample. Our situation is better described by the ballistic model introduced by Schechter et al. ${ }^{12}$ The basic features of this model can be understood in a simplified picture with only a single point of reflection at each $S / 2 \mathrm{DEG}$ interface. The transport mechanism consists of a series of normal and Andreev reflections, as illustrated in Fig. 1(c). The amplitude for Andreev reflection at the left and the right interface can be expressed by $r_{h e, 1}=i r_{a} \exp \left(i \phi_{1}\right)$ and $r_{h e, 2}=i r_{a} \exp \left(i \phi_{2}\right)$, respectively, with $r_{a}=\left|r_{h e}\right|$, the absolute value of the Andreev reflection probability. ${ }^{12}$ If we denote the amplitude for a normal reflection of an electron or a hole by $r_{e e}$ and $r_{h h}$, respectively, with $r_{e e}=r_{h h}^{*}$, we arrive at the following expression for the total Andreev reflection amplitude from the upper normal contact to the superconducting electrodes at zero bias: 


$$
r_{2 D E G / S}=i r_{a} e^{i \phi_{1}}\left(1+\frac{\left|r_{e e}\right|^{2} e^{i\left(\phi_{2}-\phi_{1}\right)}}{1-r_{a}^{2} e^{i\left(\phi_{2}-\phi_{1}\right)}}\right) .
$$

For a single channel the zero-bias conductance $G_{0}$ is given by $\left(4 e^{2} / h\right)\left|r_{2 D E G / S}\right|^{2}$, which results in

$$
G_{0}=\frac{4 e^{2}}{h} r_{a}^{2}\left(1+\left|r_{e e}\right|^{2} \frac{2 \cos \phi-2 r_{a}^{2}+\left|r_{e e}\right|^{2}}{1-2 r_{a}^{2} \cos \phi+r_{a}^{4}}\right) .
$$

In Fig. 2 (upper inset) the calculated conductance vs $\phi$ is shown exemplarily for $r_{a}^{2}=0.1$ and $\left|r_{e e}\right|^{2}=0.9$. As can be seen here, $G_{0}$ is an even function of $\phi$ with a maximum at $\phi=0$. Of course, our calculation only illustrates the principle mechanism leading to the phase dependence of $G_{0}$. The calculated curve and the experimental results are in qualitative agreement. However, the experimentally achieved modulation of $G_{0}$ is considerably smaller than the calculated one. We attribute this discrepancy to the simplified assumptions of our model.

A comparison with the more rigorous calculations by Nakano and Takayanagi ${ }^{20}$ and by Schechter et al. ${ }^{12}$ shows that the major features that are predicted are confirmed in our measurement. The experimentally observed slow decrease of $G_{0}$ with increasing $\phi$ is in close agreement to the corresponding calculation for a ballistic beam splitter. ${ }^{20}$ However, the measured increase of the zero-bias conductance is considerably lower than theoretically predicted. ${ }^{12}$ We attribute this to the fact that presumably the reflection at the interface are partially diffusive. Furthermore, although the separation of the $\mathrm{Nb}$ electrodes is smaller than the elastic mean free path, the width $W$ of the electrodes is comparable to $l_{e}$. Thus, the overall transport takes place in the intermediate regime between the ballistic and diffusive regimes.

The local phase difference between the superconducting electrodes of a junction can be controlled by applying a magnetic field normal to the plane of the 2DEG. In order to study the effect on the quasiparticle interference, the $d I / d V-I_{s}$ characteristics were measured as a function of $B$. As can be seen in Fig. 3 (inset), the range where an enhanced differential conductance is found (indicated by the lighter gray areas) shows a Fraunhofer-like pattern. This directly corresponds to the regular Fraunhofer interference pattern of the $I_{c}-B$ characteristics (not shown here), indicating that the supercurrent distribution $j(y)$ is homogeneous. At $B= \pm 0.11 \mathrm{mT}$, corresponding to a single flux quantum $\Phi_{0}$ penetrating the normal region of the junction, the $d I / d V$ characteristics shows almost no dependence on $I_{s}$.

At finite magnetic fields the differential conductance is no longer symmetric with respect to the junction bias current. For $B=+50 \mu \mathrm{T}$, the location of conductance maximum (indicated by an arrow in Fig. 3) is found at a negative junction bias current, whereas at the reversed field the maximum is found at the opposite side. The shift can be explained by having a closer look on the phase difference $\phi(y)$ along the superconducting electrodes as a function of the magnetic flux $\Phi=B A$ within the 2DEG area $A$ of the junction. In Fig. 4(a), the sinusoidal dependence of $j(y)$ and the linear increase of $\phi(y)$ for $\Phi<\Phi_{0}$ are depicted for $I_{s}=0$. If a forward

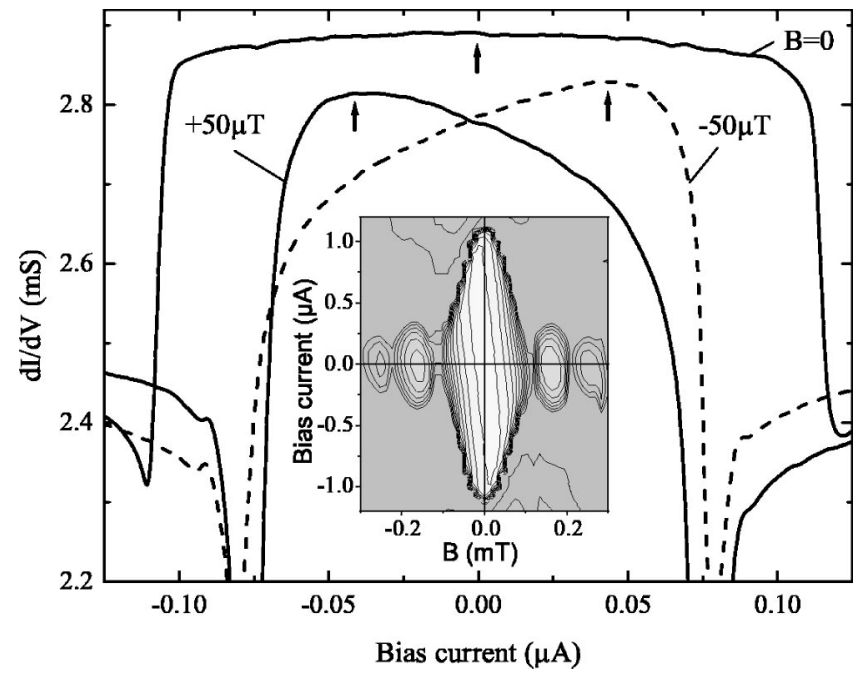

FIG. 3. Differential conductance vs junction bias current at $B$ $=0$ and $\pm 50 \mu \mathrm{T}$. The temperature was $0.6 \mathrm{~K}$. The inset shows $d I / d V$ as a function of $B$ and $I_{s}$. The conductance is represented by a gray scale.

bias current is applied $\left(I_{s}>0\right)$, the phase is shifted by a constant value $\Delta \phi$ leading to a corresponding current distribution $j(y)$ in forward direction [Fig. 4(b)]. In contrast, if a reverse bias current is applied, the phase difference is shifted by $-\Delta \phi$ and the current density is reversed [Fig. 4(c)]. It was pointed out by Schechter et al. ${ }^{12}$ that for junctions with a large electrode width, it can be expected that the relevant area for the interference effects is close to the normal electrode $(y \approx W)$. The corresponding dependencies of $\phi(W)$ on $I_{s}$ are shown schematically in Fig. 4(d) for different magnetic field orientations. At $\Phi=0$, zero phase difference and

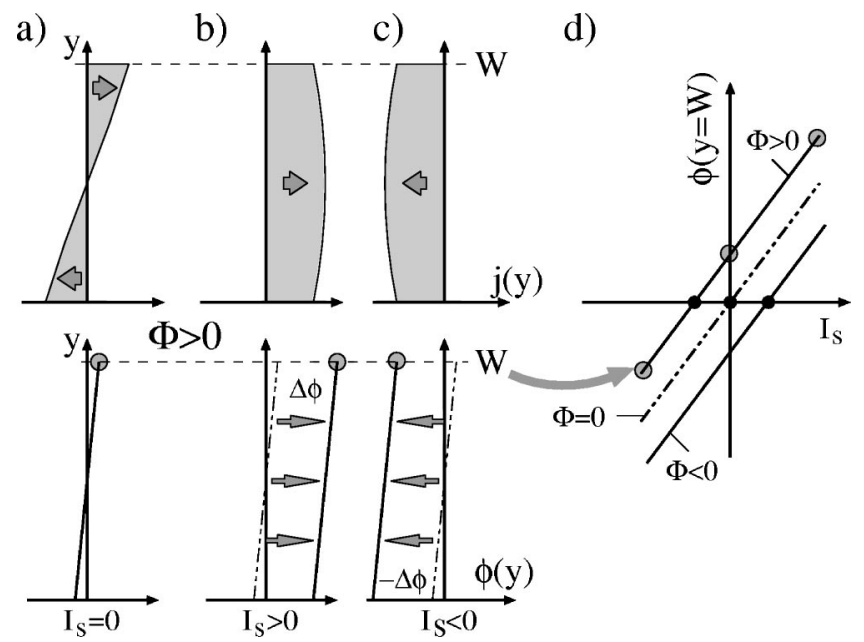

FIG. 4. Schematic illustration of the current density $j(y)$ and phase difference $\phi(y)$ along a $S / 2 \mathrm{DEG} / S$ junction at $\Phi>0$ for different bias currents: (a) $I_{s}=0$, (b) $I_{s}>0$, and (c) $I_{s}<0$. (d) Illustration of $\phi(y=W)$ as a function of $I_{s}$ for $\Phi>0, \Phi=0$, and $\Phi<0$. The gray circles represent the phase at $y=W$ for $I_{s}<0, I_{s}$ $=0$, and $I_{s}>0$ for $\Phi>0$. The black dots show at which bias current polarity zero phase difference at $y=W$ is obtained for $\Phi>0$, $\Phi=0$, and $\Phi<0$. 
thus a maximum in $d I / d V$, is found at $I_{s}=0$. Whereas, at $\Phi>0$ the phase difference at $y=W$ is shifted by $2 \pi\left(\Phi / \Phi_{0}\right)$ resulting in $\phi(W)=0$ at $I_{s}<0$. This is in agreement to the experimental findings where the maximum is found at reverse bias currents. Analogously, for $\Phi<0$, zero phase difference is obtained at $I_{s}>0$.

In order to describe the influence of the magnetic field on the interference in more detail the phase gradient along the junction electrodes and the flux encircled by the quasiparticles have to be taken into account. ${ }^{12}$ Especially at larger magnetic fields where the phase gradient along the junction is larger, it can be expected that the interference effects are averaged out. This explains the reduced conductance at larger fields.
In conclusion, we have shown that the differential conductance between a normal conductor and superconducting electrodes of a $S / 2 \mathrm{DEG} / S$ junction is controlled by interference effects. The interference effects are due to the phase difference imposed by a Josephson current. By applying an external magnetic field, the interference can be modified.

Fruitful discussions with I. E. Batov, A. Brinkman, A. A. Golubov, V. A. Guzenko, and M. Schechter are gratefully acknowledged. The authors thank A. van der Hart for the electron beam lithography and H. Kertz for his excellent assistance during the measurements. This work was supported by the Deutsche Forschungsgemeinschaft (DFG) Contract No. SCHA 835 1-2.

${ }^{11}$ H.A. Blom, A. Kadigrobov, A.M. Zagoskin, R.I. Shekhter, and M. Jonson, Phys. Rev. B 57, 9995 (1998).

*Electronic address: th.schaepers@fz-juelich.de

${ }^{\dagger}$ Present address: Infineon Technologies, P.O. Box 1009 40, 01076

Dresden, Germany.

${ }^{\ddagger}$ Present address: Global Light Industries, 47475 Kamp-Lintfort, Germany.

${ }^{1}$ T.M. Klapwijk, Physica B 197, 481 (1994).

${ }^{2}$ B. J. van Wees and H. Takayanagi, in Mesoscopic Electron Transport, edited by L. L. Son et al. (Kluwer Academic Publishers, The Netherlands, 1997), pp. 469-501.

${ }^{3}$ Th. Schäpers, Superconductor/Semiconductor Junctions, Springer Tracts on Modern Physics Vol. 174 (Springer-Verlag, Berlin, 2001).

${ }^{4}$ I.O. Kulik, Zh. Éksp. Teor. Fiz. 57, 1745 (1969) [Sov. Phys. JETP 30, 944 (1970)].

${ }^{5}$ B.Z. Spivak and D.E. Khmelnitskii, Pis'ma Zh. Éksp. Teor. Fiz. 35, 334 (1982) [JETP Lett. 35, 412 (1982)].

${ }^{6}$ C. Lambert, J. Phys.: Condens. Matter 5, 707 (1993).

${ }^{7}$ F.W.J. Hekking and Y.V. Nazarov, Phys. Rev. Lett. 71, 1625 (1993).

${ }^{8}$ V.C. Hui and C.J. Lambert, Europhys. Lett. 23, 203 (1993).

${ }^{9}$ C.W.J. Beenakker, J.A. Melsen, and P.W. Brouwer, Phys. Rev. B 51, 13883 (1995).

${ }^{10}$ N.R. Claughton, R. Raimondi, and C.J. Lambert, Phys. Rev. B 53, 9310 (1996).
${ }^{12}$ M. Schechter, Y. Imry, and Y. Levinson, Phys. Rev. B 64, 224513 (2001).

${ }^{13}$ H. Pothier, S. Guèron, D. Esteve, and M.H. Devoret, Phys. Rev. Lett. 73, 2488 (1994).

${ }^{14}$ B.J. van Wees, A. Dimoulas, J.P. Heida, T.M. Klapwijk, W. van der Graaf, and G. Borghs, Physica B 203, 285 (1994).

${ }^{15}$ V.T. Petrashov, V.N. Antonov, P. Delsing, and T. Claeson, Phys. Rev. Lett. 74, 5268 (1995).

${ }^{16}$ A. Dimoulas, J.P. Heida, B.J. van Wees, T.M. Klapwijk, W. van der Graaf, and G. Borghs, Phys. Rev. Lett. 74, 602 (1995).

${ }^{17}$ H. Courtois, Ph. Gandit, D. Mailly, and B. Pannetier, Phys. Rev. Lett. 76, 130 (1996).

${ }^{18}$ A.F. Morpurgo, B.J. van Wees, T.M. Klapwijk, and G. Borghs, Phys. Rev. Lett. 79, 4010 (1997).

${ }^{19}$ P.G.N. de Vegvar, T.A. Fulton, W.H. Mallison, and R.E. Miller, Phys. Rev. Lett. 73, 1416 (1994).

${ }^{20}$ H. Nakano and H. Takayanagi, Phys. Rev. B 47, 7986 (1993).

${ }^{21}$ Th. Schäpers, R.P. Müller, G. Crecelius, H. Hardtdegen, and H. Lüth, J. Appl. Phys. 88, 4440 (2000).

${ }^{22}$ K.K. Likharev, Rev. Mod. Phys. 51, 101 (1979).

${ }^{23}$ Th. Schäpers, A. Kaluza, K. Neurohr, J. Malindretos, G. Crecelius, A. van der Hart, H. Hardtdegen, and H. Lüth, Appl. Phys. Lett. 71, 3537 (1997). 